\title{
Implementation of earlier antibiotic administration in patients with severe sepsis and septic shock in Japan: a descriptive analysis of a prospective observational study
}

Toshikazu Abe ${ }^{1,2,3^{*}}$, Shigeki Kushimoto ${ }^{4}$, Yasuharu Tokuda ${ }^{5}$, Gary S. Phillips ${ }^{6}$, Andrew Rhodes ${ }^{7}$, Takehiro Sugiyama ${ }^{2,3,8,9}$, Akira Komori ${ }^{1}$, Hiroki Iriyama ${ }^{1}$, Hiroshi Ogura ${ }^{10}$, Seitaro Fujishima ${ }^{11}$, Atsushi Shiraishi ${ }^{12}$, Daizoh Saitoh ${ }^{13}$, Toshihiko Mayumi ${ }^{14}$, Toshio Naito ${ }^{1}$, Kiyotsugu Takuma ${ }^{15}$, Taka-aki Nakada ${ }^{16}$, Yasukazu Shiino ${ }^{17}$, Takehiko Tarui $^{18}$, Toru Hifumi ${ }^{19}$, Yasuhiro Otomo ${ }^{20}$, Kohji Okamoto ${ }^{21}$, Yutaka Umemura ${ }^{10}$, Joji Kotani ${ }^{22}$, Yuichiro Sakamoto ${ }^{23}$, Junichi Sasaki ${ }^{24}$, Shin-ichiro Shiraishi ${ }^{25}$, Ryosuke Tsuruta ${ }^{26}$, Akiyoshi Hagiwara ${ }^{27}$, Kazuma Yamakawa ${ }^{28}$, Tomohiko Masuno ${ }^{29}$, Naoshi Takeyama ${ }^{30}$, Norio Yamashita ${ }^{31}$, Hiroto lkeda ${ }^{32}$, Masashi Ueyama ${ }^{33}$, Satoshi Gando 34,35 and on behalf of JAAM FORECAST group

\begin{abstract}
Background: Time to antibiotic administration is a key element in sepsis care; however, it is difficult to implement sepsis care bundles. Additionally, sepsis is different from other emergent conditions including acute coronary syndrome, stroke, or trauma. We aimed to describe the association between time to antibiotic administration and outcomes in patients with severe sepsis and septic shock in Japan.
\end{abstract}

Methods: This prospective observational study enrolled 1184 adult patients diagnosed with severe sepsis based on the Sepsis-2 criteria and admitted to 59 intensive care units (ICUs) in Japan between January 1, 2016, and March 31, 2017, as the sepsis cohort of the Focused Outcomes Research in Emergency Care in Acute Respiratory Distress Syndrome, Sepsis and Trauma (FORECAST) study. We compared the characteristics and in-hospital mortality of patients administered with antibiotics at varying durations after sepsis recognition, i.e., 0-60, 61-120, 121-180, 181240, 241-360, and 361-1440 min, and estimated the impact of antibiotic timing on risk-adjusted in-hospital mortality using the generalized estimating equation model (GEE) with an exchangeable, within-group correlation matrix, with "hospital" as the grouping variable.

(Continued on next page)

\footnotetext{
* Correspondence: abetoshi111@gmail.com

${ }^{1}$ Department of General Medicine, Juntendo University, Tokyo, Japan

${ }^{2}$ Health Services Research and Development Center, University of Tsukuba,

Tsukuba, Japan

Full list of author information is available at the end of the article
}

(c) The Author(s). 2019 Open Access This article is distributed under the terms of the Creative Commons Attribution 4.0 International License (http://creativecommons.org/licenses/by/4.0/), which permits unrestricted use, distribution, and reproduction in any medium, provided you give appropriate credit to the original author(s) and the source, provide a link to the Creative Commons license, and indicate if changes were made. The Creative Commons Public Domain Dedication waiver (http://creativecommons.org/publicdomain/zero/1.0/) applies to the data made available in this article, unless otherwise stated. 
(Continued from previous page)

Results: Data from 1124 patients in 54 hospitals were used for analyses. Of these, 30.5\% and 73.9\% received antibiotics within $1 \mathrm{~h}$ and $3 \mathrm{~h}$, respectively. Overall, the median time to antibiotic administration was 102 min [interquartile range (IQR), 55-189]. Compared with patients diagnosed in the emergency department [90 min (IQR, 48-164 min)], time to antibiotic administration was shortest in patients diagnosed in ICUs [60 min (39-180 min)] and longest in patients transferred from wards [120 min (62-226)]. Overall crude mortality was 23.4\%, where patients in the 0-60 min group had the highest mortality (28.0\%) and a risk-adjusted mortality rate [28.7\% (95\% Cl 23.3$34.1 \%)$, whereas those in the 61-120 min group had the lowest mortality (20.2\%) and risk-adjusted mortality rates [21.6\% (95\% Cl 16.5-26.6\%)]. Differences in mortality were noted only between the 0-60 min and 61-120 min groups.

Conclusions: We could not find any association between earlier antibiotic administration and reduction in inhospital mortality in patients with severe sepsis.

Keywords: Sepsis, Antibiotic, Bundle, Protocols

\section{Key points}

- In Japan, one third of the patients received antibiotics within $1 \mathrm{~h}$ and three fourths within $3 \mathrm{~h}$ of sepsis recognition.

- Our descriptive results do not support early antibiotic administration, i.e., within $1 \mathrm{~h}$ after diagnosis, for reducing in-hospital mortality in patients with severe sepsis and septic shock.

\section{Background}

Time to antibiotic administration is a key element in sepsis care, and the Surviving Sepsis Campaign (SSC) guidelines (2004, 2008, 2012, and 2016) have repeatedly recommended initiating empirical broad-spectrum therapy within $3 \mathrm{~h}$ from triage or sepsis recognition [1-4]. However, the updated 2018 SSC guidelines recommend a 1-h window for antibiotic administration following the recognition of sepsis as a reasonable approach [5]; this update has been significantly debated, and it remains controversial [6]. Although it would be impossible to argue against appropriate and timely antibiotic therapy for sepsis considering its time-sensitive nature, sepsis is different from other emergent conditions such as acute coronary syndrome, stroke, or trauma. Specifically, its recognition by healthcare providers within $1 \mathrm{~h}$ of presentation may be difficult to achieve in real-life settings because of vague presenting symptoms and the fact that its exact onset is mostly unobservable. Further, the only randomized controlled trial (RCT) that evaluated early antibiotic use in patients with suspected infection failed to reduce mortality, although this was in the prehospital setting [7].

We prospectively evaluated the characteristics and management of patients with severe sepsis in the Focused Outcomes Research in Emergency Care in Acute Respiratory Distress Syndrome, Sepsis, and Trauma (FORECAST) study in Japan [8]. We then used the
FORECAST database to describe the association between time to antibiotic administration and outcomes in patients with severe sepsis and septic shock treated in real-world clinical settings.

\section{Methods}

The study protocol was reviewed and approved by the ethics committee of all participating institutions in the Japanese Association for Acute Medicine (JAAM) study group, Japan (IRB No.014-0306 on Hokkaido University, a representative institution for FORECAST). Obtaining an informed consent from a study participant was waived under the approval of the ethics committees.

\section{Design and setting}

This is a predefined secondary analysis of the FORECAST study. We predefined the secondary analyses when we set variables of the FORECAST study. It selected a cohort of patients with severe sepsis and septic shock who were registered in the FORECAST study, which was a multi-center, prospective data collection study on acutely ill patients that included those with acute respiratory distress syndrome, sepsis, and trauma. The FORECAST study obtained data from patients admitted to 59 intensive care units (ICUs) in Japan and was conducted from January 1, 2016, to March 31, 2017.

\section{Participants}

We included adult patients ( $\geq 16$ years) diagnosed with severe sepsis and septic shock based on the Sepsis-2 criteria published in 2003 [9] and admitted to the ICU. The exclusion criteria were unfavorable sustained lifecare or post-cardiopulmonary arrest resuscitation status at the time of sepsis recognition, missing data on antibiotic timing or in-hospital mortality, or time to antibiotic use $>1440 \mathrm{~min}$. 


\section{Data collection}

Relevant patient data, originally compiled by the FORECAST investigators, were obtained from the FORECAST database. Hospital information obtained included number, specialty, type of facility and staff, number of patients, and number of beds. Patient data, collected as part of the clinical workup, included demographic characteristics of patients, organ dysfunctions, sepsis-related severity scores, time to antibiotic administration, inhospital mortality, 28-day mortality, ICU-free days, ventilator-free days (VFD), and length of hospital stay. Additionally, we obtained data on compliance with established sepsis care bundles, such as measurement of the initial serum lactate levels within $3 \mathrm{~h}$.

\section{Data definitions}

Sepsis care bundles were defined according to the SSC guidelines (2012) [3] as to whether all bundle elements were achieved within the appropriate time frame (i.e., 3 or $6 \mathrm{~h}$ ) and if they adhered to indications (i.e., septic shock or lactate level $>4 \mathrm{mmol} / \mathrm{L}$ ). For all patients, protocol initiation time was defined as the time of sepsis recognition at the emergency department (ED), ward, or ICU. Sepsis recognition was a clinical judgment, wherein a physician-in-charge had suspected sepsis at the initial evaluation. Timestamp was recorded in the database by a physician-in-charge. Patients with antibiotic before arrival were recognized as patients with infection but not sepsis. After arrival, time would start when a physicianin-charge recognized a patient with sepsis. Time from sepsis recognition to initiation of antibiotics (time to antibiotic) was divided into six groups defined as $0-60$, 61-120, 121-180, 181-240, 241-360, and 361-1440 min. Septic shock was defined based on the Sepsis-2 criteria [9], and VFD was defined as the number of days within the first 28 days after hospital admission during which a patient was able to breathe without a ventilator. VFD for patients who died during the study period was listed as 0 . ICU-free days were similarly calculated.

\section{Analysis}

The primary outcome was in-hospital mortality. Secondary outcomes were VFD and ICU-free days. The exposure was time to antibiotic. Because the missing data were low, no assumptions were made for this factor.

Descriptive statistics included frequency and percentage for categorical variables and mean \pm standard deviation $(\mathrm{SD})$ or medians and interquartile range (IQR) for continuous variables, as appropriate. We compared baseline characteristics and outcomes among patients with severe sepsis in the six time to antibiotic therapy groups (0-60, 61-120, 121-180, 181-240, 241-360, and 361$1440 \mathrm{~min}$ ) using analysis of variance, Kruskal-Wallis test or chi-square test, as required.
The impact of antibiotic timing on risk-adjusted hospital mortality was estimated using the generalized estimating equation (GEE) model with an exchangeable within-group correlation matrix where the hospital was the panel or grouping variable. The following covariates were specified a priori based on clinical experience and prior studies: patient age, gender, admission source (ED, ward, or in ICU), Charlson comorbidity index (CCI), antibiotic use before arrival, site of infection (e.g., lung, abdomen, urinary tract, soft tissue, central nerve system, or blood stream-related), sepsis-related organ failure assessment (SOFA) score, and intravenous fluid bolus completed within $3 \mathrm{~h}(30 \mathrm{mg} / \mathrm{kg}$ crystalloid). We also performed the same analysis after replacing time to antibiotic as a continuous variable. In a subgroup analysis, we stratified patients with septic shock and those from only ED based on hospital admission source and analyzed these subgroups as described in the primary analysis [i.e., the GEE model adjusted patient age, gender, $\mathrm{CCI}$, antibiotic use before arrival, site of infection, SOFA score, and intravenous fluid bolus completed within $3 \mathrm{~h}$ (30 mg/kg crystalloid)].

All statistical analyses were performed using Stata software version 15.1 (StataCorp, TX, USA).

\section{Results}

We recruited 1184 patients with severe sepsis who were admitted to ICUs at participating institutions during the FORECAST study period. Of these, 60 patients were excluded because of missing data on the timing of antibiotic administration $(n=11)$ or in-hospital mortality $(n=33)$ or because the time to antibiotic was greater than $1440 \mathrm{~min}$. Of the remaining 1124 participants who were admitted to 54 hospitals, $30.5 \%$ of the patients received antibiotics within $1 \mathrm{~h}$, and $73.9 \%$ of the patients received antibiotics within $3 \mathrm{~h}$. Overall, the median time to antibiotic administration was $102 \mathrm{~min}$ (IQR, 55-189), and compared with patients diagnosed in the ED [90 min (IQR, 48-164 min)], time to antibiotic administration was shortest in patients diagnosed in ICUs $[60 \mathrm{~min}$ (39-180 $\mathrm{min})]$ and longest in patients who had been transferred from wards [120 min (62-226 min)], implying that patients diagnosed in the ED or the ICU received antibiotics earlier than those in the ward.

Baseline characteristics, categorized based on the timing of antibiotic administration, are detailed in Table 1. The $0-60,61-120$, and $121-180 \mathrm{~min}$ groups received lower pre-antibiotic therapy, such as oral medicines for infection, than the other groups. The $0-60,61-120$, and 121-180 min groups were also more likely to achieve the 3-h bundle, such as obtaining blood cultures and intravenous fluid bolus than the other groups.

Overall crude mortality was $23.4 \%$ in the study population. Crude mortalities were $27.7 \%$ and $16.2 \%$ among 


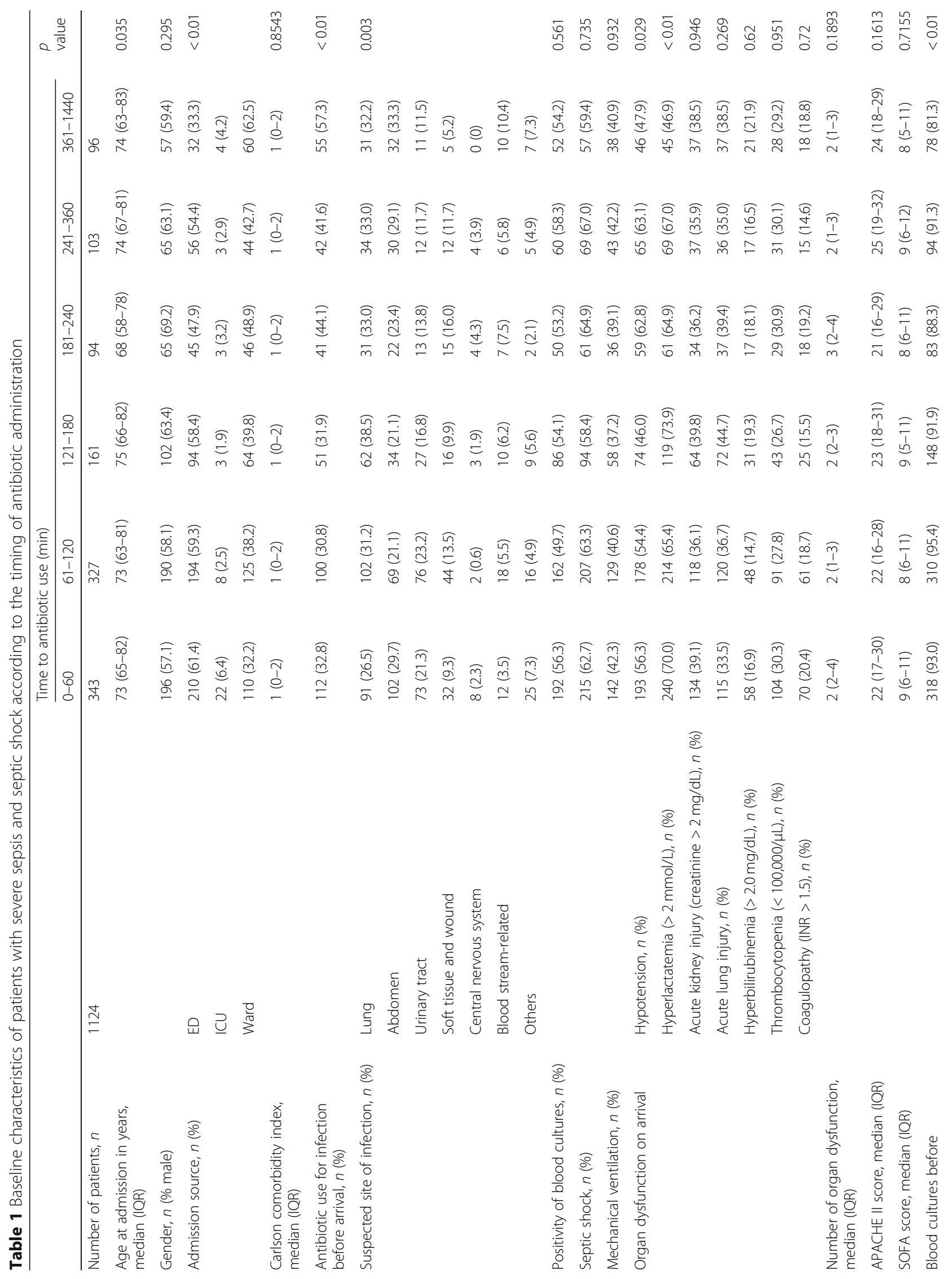




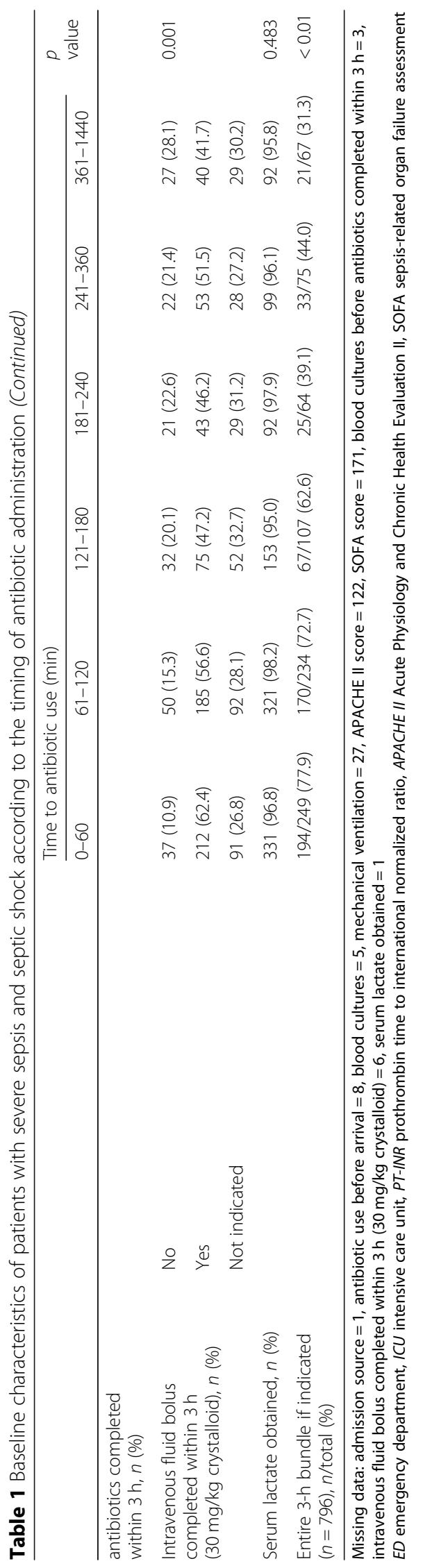


patients with and without shock, respectively. Patients received antibiotics within the first $60 \mathrm{~min}$ had the highest rates of mortality $(28.0 \%)$ and risk-adjusted mortality [28.7\% (95\% CI 23.3-34.1); Table 2, Fig. 1], whereas that of patients administered with antibiotics between 61 and $120 \mathrm{~min}$ had the lowest (20.2\%) and risk-adjusted mortality rates $[21.6 \%$ (95\% CI 16.5-26.6)]. Importantly, mortality was different only between patients in the 0 60 and 61-120 min groups. In addition, time to antibiotics as a continuous variable was not related to mortality, either (odds ratio 0.999 [0.997-1.000; $P=0.152]$ ). Subgroup analysis yielded a crude mortality rate of $27.7 \%$ in patients with septic shock, and patients in the 0-60 min group continued to have the highest mortality $(31.6 \%)$ and risk-adjusted mortality rates [30.0\% (95\% CI 23.5-36.5)], whereas those patients administered with antibiotics between 361 and $1440 \mathrm{~min}$ were the lowest [21.6\% (95\% CI 11.1-32.0)]. In addition, the subgroup analysis yielded a crude mortality rate of $21.9 \%$ in the ED patients, and patients in the 0-60 min group continued to have the highest mortality (29.1\%) and riskadjusted mortality rates (27.9\% [95\% CI 21.1-34.6]), whereas that in patients administered with antibiotics between 361 and $1440 \mathrm{~min}$ was the lowest [12.8\% (95\% CI 5.7-25.0)].

\section{Discussion}

\section{Summary}

In emergency medical centers in Japan, one third of patients receive antibiotics within $1 \mathrm{~h}$ and three quarters within $3 \mathrm{~h}$ of sepsis recognition. Although we have found high levels of adherence to the sepsis care bundle (2012) during the study period, we were unable to show a linear relationship between the timing of antibiotic administration, such as within $1 \mathrm{~h}$ or $3 \mathrm{~h}$ after sepsis recognition, and in-hospital mortality among patients with severe sepsis and septic shock.

\section{Comparison with previous studies}

Although previous studies have shown that a delay in antibiotic administration is associated with a higher inhospital mortality rate [10-14], our findings from a prospective observational study obtained data of antibiotic administration timing do not support their results. Neither prospective nor retrospective observational data can establish causation, and discrepancies between these results only raise further questions. Interestingly, a metaanalysis of studies regarding the relationship between antibiotic administration timing and mortality found no significant benefit after administering antibiotics within $1-3 \mathrm{~h}$ from triage or sepsis recognition in comparison with administration at a later timing [15], and a RCT of providing antibiotics to patients with suspected sepsis in the ambulance did not show improved survival [7].
Nonetheless, these previous studies [7, 10-13] and the present study have three major problems: (1) understanding the clinical courses of sepsis, including its onset; (2) stratification of sepsis severity and cognate adjustments to treatment strategies, e.g., rapid treatments; and (3) establishing correlation or causation in sepsis care bundle studies.

Sepsis is different from other time-dependent and emergent conditions such as acute coronary syndrome because its precise onset is difficult to establish and the lack of a specific diagnostic marker makes recognition problematic. Three phases in the clinical course of sepsis are recognized: (A) time from onset of infection to a detectable condition, (B) time from a detectable condition to diagnosis, and $(\mathrm{C})$ time from diagnosis to antibiotic administration (time to antibiotic), and although phases A and B are significantly shorter in other timedependent emergent conditions, the duration of these two phases varies considerably in sepsis. Furthermore, it is difficult to focus only on phase $\mathrm{C}$ to reduce mortality in sepsis as other variables that can affect outcomes include the timestamp of antibiotic initiation (time zero) and patient location, as seen in previous studies [15] and in our study. Similar to other studies, we defined time zero as the time of sepsis recognition, although it is a subjective choice [15], because the clinical course of each patient would differ based on the presenting symptoms, even if triage at ED is chosen as a reliable time zero point [16]. Thus, detection of sepsis and antibiotic initiation are equally important as delays in appropriate antibiotic therapy are more likely to occur in patients with complex or atypical presentations who are also more likely to harbor drug-resistant organisms [17]. Moreover, reports show that inter-hospital transfer delayed the administration of initial antibiotics, although prognosis was not different [18]. Similar to previous reports, our patients included not only those presenting to ED but also those being transferred from other hospitals [15], and it is essential to disentangle such complicated definitions of time zero and locations in each study before direct comparisons can be made among these studies.

Sepsis severity stratification and adjustment with rapid treatment may have been insufficient in previous and our studies regarding time to antibiotic, and conflating sepsis and septic shock may also be a confounder. Although $63 \%$ of the patients in our study presented with shock, we could not find any association between earlier time to antibiotic and reduced mortality in all participants. Recent studies have demonstrated a weak association between time to antibiotic therapy and mortality in all cohorts (a relatively mild state in comparison with our cohort), although a large significant association between delays in antibiotic administration and higher 


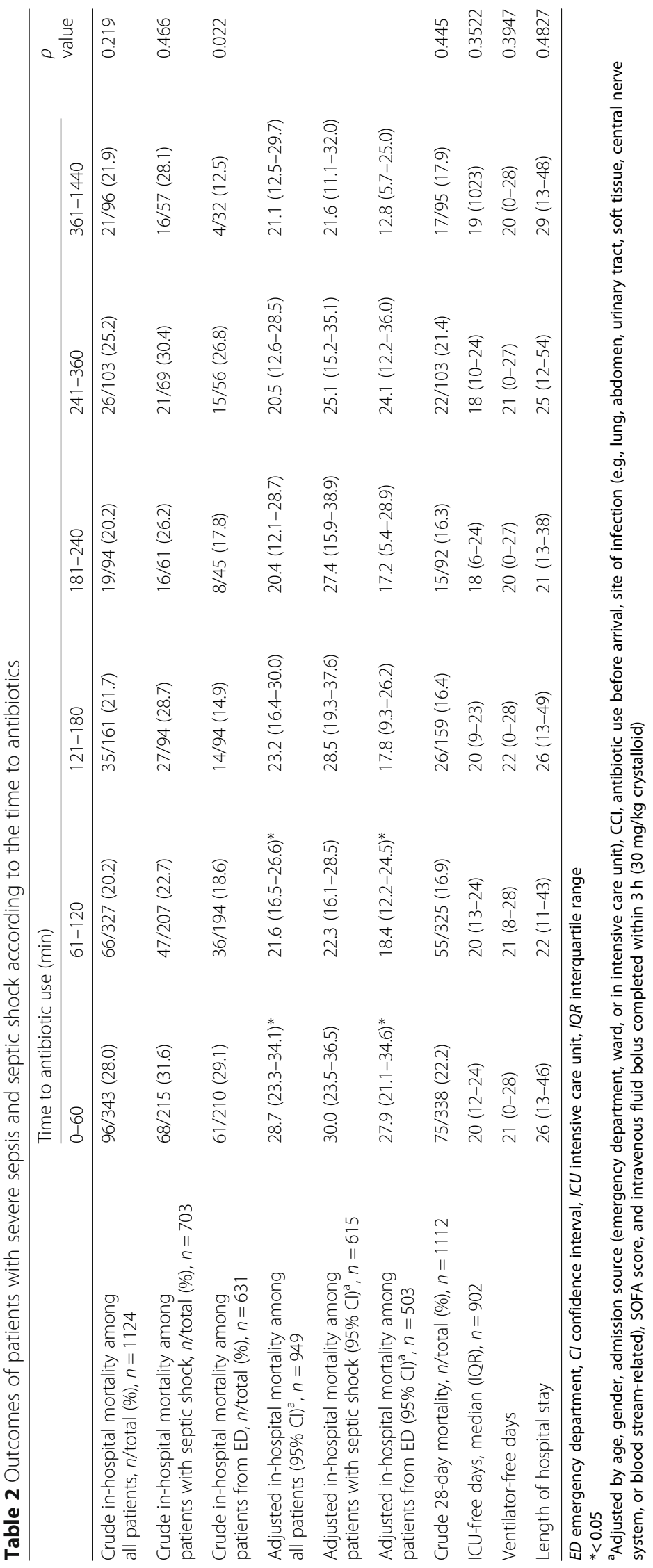




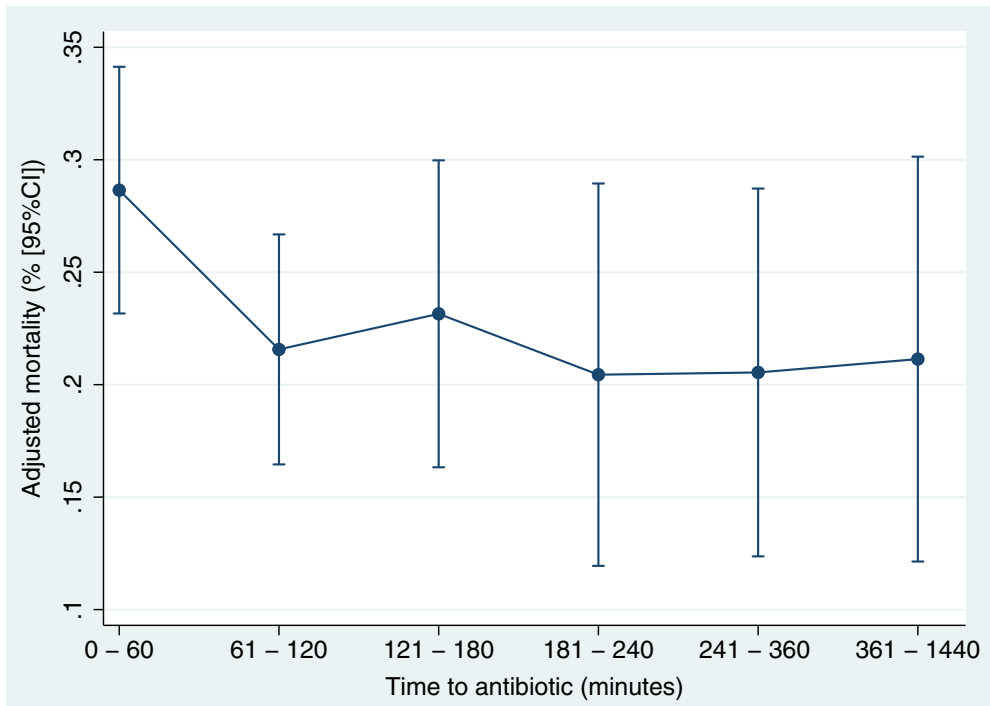

Fig. 1 Adjusted in-hospital mortality according to the timing of antibiotic administration among patients with severe sepsis and septic shock. Adjusted by age, gender, admission source (emergency department, ward, or in intensive care unit), CCl, antibiotic use before arrival, site of infection (e.g., lung, abdomen, urinary tract, soft tissue, central nerve system, or blood stream-related), SOFA score, and intravenous fluid bolus completed within $3 \mathrm{~h}$ (30 mg/kg crystalloid)

mortality rates in patients with septic shock [10, 11]. Further, an RCT of administering antibiotics to patients with suspected sepsis in the ambulance also included many patients without shock [7]. In the study, it notes that even patients in the control group received antibiotics quite early and that the patients were treated with low risk (mortality rate $8 \%$ ). This is quite a different population compared with that in the hospital setting. Indeed, severity adjustment is difficult in sepsis studies because sepsis includes complicated etiologies, various presentations, and severities compared with other medical emergencies [19]. Nevertheless, in our study, we used fewer variables to adjust severity in comparison with previous studies owing to the limitations of our database $[12,13]$. Previous studies have reported higher crude mortality rates in patients administered with antibiotics in the first hour, which then declined over time; however, severity adjustment reversed the relationship between time to antibiotic and mortality $[12,13]$. Our study may also have been under-adjusted for severity because the sample size was relatively smaller and covariates were fewer than those in previous studies [10-13].

Next, we must consider whether this relationship between time to antibiotic and mortality causative or only a correlation, given that all other components of the sepsis care bundle may be potentially physiologically effective along with time to antibiotic and that the effectiveness of each component remains controversial in various settings $[10,20,21]$. Adherence to the sepsis care bundle also varies in each setting $[8,10,22,23]$. For example, in a study among patients who received antibiotics as they met the criteria for severe sepsis, a relatively high compliance rate for the sepsis care bundle was reported if sepsis was definitively diagnosed (with diagnosis code) compared with undiagnosed sepsis (without diagnosis code) [22]. In our study, the $0-60$, 61-120, and 121-180 min groups were more likely to comply with the 3-h bundle, compared with the others. Conversely, the prognosis may have been good in patients who had been diagnosed early and in those who were administered with antibiotics early [10-13], implying that diagnosing sepsis may be more important than initiating the sepsis care bundle. Further, all components of the bundle should be investigated in future studies that focus on the relationship between time to antibiotic and trigger of initiation.

\section{Possible explanations and implications}

Medical staff intuitively understands that the early use of appropriate antibiotics is an important modifiable factor. However, optimal timing and its effectiveness remain unclear after a widespread implementation of the sepsis protocol in developed countries such as Japan. Although there is no doubt that sepsis care bundle components, such as time to antibiotic, play a role in the arising importance of treatment speed, a similar situation arose when early goal-directed therapy was debated [24, 25].

In this study, patients who received antibiotics within the first hour had the highest mortality despite the greatest compliance with the entire 3-h bundle. This may be because of the severity at presentation, detecting antibiotic use within the first hour, or an obvious septic 
state. Alternatively, very early antibiotic use (within $1 \mathrm{~h}$ ) may lead to unfavorable outcomes; although, this does not seem likely as all the patients would have had a sepsis state for some time before the point of recognition [26]. Furthermore, indiscriminate and rapid use of broad-spectrum antibiotics in all patients may be potentially harmful [27, 28], as it may lead to adverse events; however, there are only few reports on adverse events of rapid use of antibiotics. Incorrect diagnosis of sepsis may lead to a delay in administering other useful treatments or appropriate source control despite the use of broad-spectrum antibiotics [17].

Although there may be a linear relationship between early time to antibiotic therapy and better prognosis in sepsis, it is presumably weak. Moreover, similar to the previous study report, our results showed that earlier time to antibiotic therapy was related to better outcomes in ED patients [14] when we excluded patients who received antibiotics within $1 \mathrm{~h}$. Otherwise, focusing on the rapid use of antibiotics may lead to misdiagnosis of the site of infection, which is related to poor outcomes [28]. Sepsis treatment and care involve components other than the time to antibiotic, and while this does not mean that rapid treatments are not good, most physicians intuitively recognize the need for it. Thus, timely antibiotic administration to patients with sepsis should remain one of the key elements in the sepsis care bundle, regardless of time restriction.

\section{Limitations}

This study has several limitations. First, controlling for confounders may have been insufficient, as there are potential unknown confounders not available to us. Antibiotic initiation can be determined not only based on the patient's severity but also on unknown factors that are difficult to be quantified. Second, there were patients who were using antibiotics before arrival. We have adjusted antibiotic use before arrival as a covariate in the GEE models. Moreover, the results did not change significantly even if patients with antibiotic usage before arrival were excluded. Third, there may be an indication bias as antibiotics may have been prescribed within the first hour only in patients with severe (non-survivable) presentation. However, we found that the results did not change even if patients who died within 1, 2, or 3 days were excluded from the analysis, suggesting that it may be difficult to show differences in practice quality between patients presenting with considerably mild and considerably severe symptoms [7]. Forth, there may have been a social desirability bias, as physicians usually wish to be evaluated as having responded to critical patients first. Fifth, there may have been a ceiling effect with respect to severity scores, because our study population was more severely ill than those in previous studies.
Sixth, the timestamp of protocol initiation was defined as at the time of sepsis recognition, which is relatively later than the time of triage at ED. Our study may have only shown a relationship between time to antibiotic and in-hospital mortality if sepsis recognition occurred early in the course of the disease. Seventh, we did not have data about the appropriateness of antibiotic. However, as the first FORECAST paper [8] showed, $84 \%$ of patients received broad-spectrum antibiotics within $3 \mathrm{~h}$. Most patients at least adhered to empiric antibiotics based on the guideline. In addition, carbapenem was most commonly used after initial diagnoses (55\%) followed by tazobactam/piperacillin $(21 \%)$ and vancomycin (18\%). Thus, the important pathogens would have been covered by the chosen antibiotics even if antibioticresistant pathogens were present. Finally, the descriptive nature of the study could not completely identify the causal inference between the observed time to antibiotic and in-hospital mortality. In this study, we described sepsis care and implementation of sepsis bundle in Japan as one of the high adherence countries of the sepsis guideline. Our results suggested that this relationship is still controversial, and RCT of this topic should be studied further.

\section{Conclusions}

Our prospective study failed to show a difference in inhospital mortality based on the timing of antibiotic administration. Future studies are needed to prove or refute these results among patients with sepsis or septic shock.

\section{Abbreviations}

ICU: Intensive care unit; FORECAST: Focused Outcomes Research in Emergency Care in Acute Respiratory Distress Syndrome, Sepsis, and Trauma; GEE: Generalized estimating equation model; SSC: Surviving Sepsis

Campaign; RCT: Randomized controlled trial; JAAM: Japanese Association for Acute Medicine; VFD: Ventilator-free days; ED: Emergency department; SD: Standard deviation; IQR: Medians and interquartile range; CCl: Charlson comorbidity index; SOFA: Sepsis-related organ failure assessment

\section{Acknowledgements}

We thank the JAAM FORECAST Study Group for its valuable contribution to this study. We thank Enago (https://www.enago.jp) for the final English language editing. We will present this research at the 32nd Annual Congress European Society of Intensive Care Medicine (ESICM) 2019.

The following are the investigators of the JAAM FORECAST Study Group: Nagasaki University Hospital (Osamu Tasaki), Osaka City University Hospital (Yasumitsu Mizobata), Tokyobay Urayasu Ichikawa Medical Center (Hiraku Funakoshi), Aso lizuka Hospital (Toshiro Okuyama), Tomei Atsugi Hospital (Iwao Yamashita), Hiratsuka City Hospital (Toshio Kanai), National Hospital Organization Sendai Medical Center (Yasuo Yamada), Ehime University Hospital (Mayuki Aibiki), Okayama University Hospital (Keiji Sato), Tokuyama Central Hospital (Susumu Yamashita), Fukuyama City Hospital (Susumu Yamashita), JA Hiroshima General Hospital (Kenichi Yoshida), Kumamoto University Hospital (Shunji Kasaoka), Hachinohe City Hospital (Akihide Kon), Osaka City General Hospital (Hiroshi Rinka), National Hospital Organization Disaster Medical Center (Hiroshi Kato), University of Toyama (Hiroshi

Okudera), Sapporo Medical University (Eichi Narimatsu), Okayama Saiseikai General Hospital (Toshifumi Fujiwara), Juntendo University Nerima Hospital (Manabu Sugita), National Hospital Organization Hokkaido Medical Center (Yasuo Shichinohe), Akita University Hospital (Hajime Nakae), Japanese Red Cross Society Kyoto Daini Hospital (Ryouji liduka), Maebashi Red Cross 
Hospital (Mitsunobu Nakamura), Sendai City Hospital (Yuji Murata), Subaru Health Insurance Society Ota Memorial Hospital (Yoshitake Sato), Fukuoka University Hospital (Hiroyasu Ishikura), Ishikawa Prefectural Central Hospital (Yasuhiro Myojo), Shiga University of Medical Science (Yasuyuki Tsujita), Nihon University School of Medicine (Kosaku Kinoshita), Seirei Yokohama General Hospital (Hiroyuki Yamaguchi), National Hospital Organization Kumamoto Medical Center (Toshihiro Sakurai), Saiseikai Utsunomiya Hospital (Satoru Miyatake), National Hospital Organization Higashi-Ohmi General Medical Center (Takao Saotome), National Hospital Organization Mito Medical Center (Susumu Yasuda), Tsukuba Medical Center Hospital (Toshikazu Abe), Osaka University Graduate School of Medicine (Hiroshi Ogura, Yutaka Umemura), Kameda Medical Center (Atsushi Shiraishi), Tohoku University Graduate School of Medicine (Shigeki Kushimoto), National Defense Medical College (Daizoh Saitoh), Keio University School of Medicine (Seitaro Fujishima, Junichi Sasaki), University of Occupational and Environmental Health (Toshihiko Mayumi), Kawasaki Medical School (Yasukazu Shiino), Chiba University Graduate School of Medicine (Taka-aki Nakada), Kyorin University School of Medicine (Takehiko Tarui), Kagawa University Hospital (Toru Hifumi), Tokyo Medical and Dental University (Yasuhiro Otomo), Hyogo College of Medicine (Joji Kotani), Saga University Hospital (Yuichiro Sakamoto), Aizu Chuo Hospital (Shin-ichiro Shiraishi), Kawasaki Municipal Kawasaki Hospital (Kiyotsugu Takuma), Yamaguchi University Hospital (Ryosuke Tsuruta), Center Hospital of the National Center for Global Health and Medicine (Akiyoshi Hagiwara), Osaka General Medical Center (Kazuma Yamakawa), Aichi Medical University Hospital (Naoshi Takeyama), Kurume University Hospital (Norio Yamashita), Teikyo University School of Medicine (Hiroto Ikeda), Rinku General Medical Center (Yasuaki Mizushima), and Hokkaido University Graduate School of Medicine (Satoshi Gando).

\section{Authors' contributions}

TA contributed to the acquisition of data, conceived and designed this study, interpreted the data, drafted the manuscript, and revised the manuscript for important intellectual content. YT, GSP, AR, TS, AK, and HI conceived and designed this study, interpreted the data, and revised the manuscript for important intellectual content. AS contributed to the acquisition of data, conducted the data cleaning, interpreted the data, and revised the manuscript for important intellectual content. SK, HO, DS, SF, TM, and SG contributed to the acquisition of data, jointly conceived and designed this study, interpreted the data, and revised the manuscript for important intellectual content. All of the authors contributed to the acquisition of the data and reviewed, discussed, and approved the final manuscript.

\section{Funding}

This study was supported by the Japanese Association for Acute Medicine (2014-01).

\section{Availability of data and materials}

The datasets analyzed during the current study are available with the corresponding author on reasonable request.

\section{Ethics approval and consent to participate}

The study protocol was reviewed and approved by the ethics committee of all participating institutes in the Japanese Association for Acute Medicine (JAAM) study group, Japan (IRB No.014-0306 on Hokkaido University, the representative for FORECAST).

\section{Consent for publication}

Not applicable

\section{Competing interests}

The authors declare that they have no competing interests.

\section{Author details}

${ }^{1}$ Department of General Medicine, Juntendo University, Tokyo, Japan. ${ }^{2}$ Health Services Research and Development Center, University of Tsukuba, Tsukuba, Japan. ${ }^{3}$ Department of Health Services Research, Faculty of Medicine, University of Tsukuba, Tsukuba, Japan. ${ }^{4}$ Division of Emergency and Critical Care Medicine, Tohoku University Graduate School of Medicine, Sendai, Japan. ${ }^{5}$ Department of Medicine, Muribushi Project for Okinawa Residency Programs, Urasoe, Japan. ${ }^{6}$ Department of Biomedical Informatics, Ohio State
University, Columbus, OH, USA. Department of Intensive Care Medicine, St George's University Hospitals Foundation Trust, London, UK. ${ }^{8}$ Diabetes and Metabolism Information Center, Research Institute, National Center for Global Health and Medicine, Tokyo, Japan. ${ }^{9}$ Department of Public Health/Health Policy, Graduate School of Medicine, The University of Tokyo, Tokyo, Japan. ${ }^{10}$ Department of Traumatology and Acute Critical Medicine, Osaka University Graduate School of Medicine, Osaka, Japan. ${ }^{11}$ Center for General Medicine Education, Keio University School of Medicine, Tokyo, Japan. ${ }^{12}$ Emergency and Trauma Center, Kameda Medical Center, Kamogawa, Japan. ${ }^{13}$ Division of Traumatology, Research Institute, National Defense Medical College, Tokorozawa, Japan. ${ }^{14}$ Department of Emergency Medicine, School of Medicine, University of Occupational and Environmental Health, Kitakyushu, Japan. ${ }^{15}$ Emergency \& Critical Care Center, Kawasaki Municipal Kawasaki Hospital, Kawasaki, Japan. ${ }^{16}$ Department of Emergency and Critical Care Medicine, Chiba University Graduate School of Medicine, Chiba, Japan. ${ }^{17}$ Department of Acute Medicine, Kawasaki Medical School, Kurashiki, Japan. ${ }^{18}$ Department of Trauma and Critical Care Medicine, Kyorin University School of Medicine, Tokyo, Japan. ${ }^{19}$ Department of Emergency and Critical Care Medicine, St. Luke's International Hospital, Tokyo, Japan. ${ }^{20}$ Trauma and Acute Critical Care Center, Medical Hospital, Tokyo Medical and Dental University, Tokyo, Japan. ${ }^{21}$ Department of Surgery, Center for Gastroenterology and Liver Disease, Kitakyushu City Yahata Hospital, Kitakyushu, Japan.

${ }^{22}$ Department of Disaster and Emergency Medicine, Kobe University Graduate School of Medicine, Kobe, Japan. ${ }^{23}$ Emergency and Critical Care Medicine, Saga University Hospital, Saga, Japan. ${ }^{24}$ Department of Emergency and Critical Care Medicine, Keio University School of Medicine, Tokyo, Japan. ${ }^{25}$ Department of Emergency and Critical Care Medicine, Aizu Chuo Hospital, Aizuwakamatsu, Japan. ${ }^{26}$ Advanced Medical Emergency \& Critical Care Center, Yamaguchi University Hospital, Ube, Japan. ${ }^{27}$ Department of Emergency Medicine, Niizashiki Chuo General Hospital, Niiza, Japan. ${ }^{28}$ Division of Trauma and Surgical Critical Care, Osaka General Medical Center, Osaka, Japan. ${ }^{29}$ Department of Emergency and Critical Care Medicine, Nippon Medical School, Tokyo, Japan. ${ }^{30}$ Advanced Critical Care Center, Aichi Medical University Hospital, Nagakute, Japan. ${ }^{31}$ Advanced Emergency Medical Service Center, Kurume University Hospital, Kurume, Japan.

${ }^{32}$ Department of Emergency Medicine, Teikyo University School of Medicine, Tokyo, Japan. ${ }^{33}$ Department of Trauma, Critical Care Medicine, and Burn Center, Japan Community Healthcare Organization, Chukyo Hospital, Nagoya, Japan. ${ }^{34}$ Division of Acute and Critical Care Medicine, Hokkaido University Graduate School of Medicine, Sapporo, Japan. ${ }^{35}$ Department of Acute and Critical Care Medicine, Sapporo Higashi Tokushukai Hospital, Sapporo, Japan.

Received: 2 August 2019 Accepted: 9 October 2019 Published online: 19 November 2019

\section{References}

1. Dellinger RP, Carlet JM, Masur H, Gerlach H, Calandra T, Cohen J, et al. Surviving Sepsis Campaign guidelines for management of severe sepsis and septic shock. Crit Care Med. 2004;32:858-73.

2. Dellinger RP, Levy MM, Carlet JM, Bion J, Parker MM, Jaeschke R, et al. Surviving Sepsis Campaign: international guidelines for management of severe sepsis and septic shock. Crit Care Med. 2008;36:296-327.

3. Dellinger RP, Levy MM, Rhodes A, Annane D, Gerlach H, Opal SM, et al. Surviving Sepsis Campaign: international guidelines for management of severe sepsis and septic shock. Crit Care Med. 2013;41:580-637.

4. Rhodes A, Evans LE, Alhazzani W, Levy MM, Antonelli M, Ferrer R, et al. Surviving Sepsis Campaign: international guidelines for management of sepsis and septic shock. Intensive Care Med. 2017:43:304-77.

5. Levy MM, Evans LE, Rhodes A. The Surviving Sepsis Campaign bundle: 2018 update. Intensive Care Med. 2018;46:997-1000.

6. Spiegel R, Farkas JD, Rola P, Kenny JE, Olusanya S, Marik PE, et al. The 2018 Surviving Sepsis Campaign's treatment bundle: when guidelines outpace the evidence supporting their use. Ann Emerg Med. 2019;73:356-8.

7. Alam N, Oskam E, Stassen PM, Exter PV, van de Ven PM, Haak HR, et al. Prehospital antibiotics in the ambulance for sepsis: a multicentre, open label, randomised trial. Lancet Respir Med. 2018;6:40-50.

8. Abe T, Ogura H, Shiraishi A, Kushimoto S, Saitoh D, Fujishima S, et al. Characteristics, management, and in-hospital mortality among patients with severe sepsis in intensive care units in Japan: the FORECAST study. Crit Care. 2018;22:322. 
9. Levy MM, Fink MP, Marshall JC, Abraham E, Angus D, Cook D, et al. 2001 SCCM/ESICM/ACCP/ATS/SIS International Sepsis Definitions Conference. Crit Care Med. 2003;31:1250-6.

10. Seymour CW, Gesten F, Prescott HC, Friedrich ME, Iwashyna TJ, Phillips GS, et al. Time to treatment and mortality during mandated emergency care for sepsis. N Engl J Med. 2017;376:2235-44.

11. Liu VX, Fielding-Singh V, Greene JD, Baker JM, Iwashyna TJ, Bhattacharya J, et al. The timing of early antibiotics and hospital mortality in sepsis. Am J Respir Crit Care Med. 2017;196:856-63.

12. Ferrer R, Martin-Loeches I, Phillips G, Osborn TM, Townsend S, Dellinger RP, et al. Empiric antibiotic treatment reduces mortality in severe sepsis and septic shock from the first hour: results from a guideline-based performance improvement program. Crit Care Med. 2014;42:1749-55.

13. Evans IVR, Phillips GS, Alpern ER, Angus DC, Friedrich ME, Kissoon N, et al. Association between the New York sepsis care mandate and in-hospital mortality for pediatric sepsis. JAMA. 2018;320:358-67.

14. Peltan ID, Brown SM, Bledsoe JR, Sorensen J, Samore MH, Allen TL, et al. ED door-to-antibiotic time and long-term mortality in sepsis. Chest. 2019;155:938-46.

15. Sterling SA, Miller WR, Pryor J, Puskarich MA, Jones AE. The impact of timing of antibiotics on outcomes in severe sepsis and septic shock: a systematic review and meta-analysis. Crit Care Med. 2015:43:1907-15.

16. Filbin MR, Lynch J, Gillingham TD, Thorsen JE, Pasakarnis CL, Nepal S, et al. Presenting symptoms independently predict mortality in septic shock: importance of a previously unmeasured confounder. Crit Care Med. 2018:46:1592-9.

17. Klompas M, Calandra T, Singer M. Antibiotics for sepsis-finding the equilibrium. JAMA. 2018;320:1433-4.

18. Faine BA, Noack JM, Wong T, Messerly JT, Ahmed A, Fuller BM, et al. Interhospital transfer delays appropriate treatment for patients with severe sepsis and septic shock: a retrospective cohort study. Crit Care Med. 2015; 43:2589-96.

19. Abe T, Ogura H, Kushimoto S, Shiraishi A, Sugiyama T, Deshpande GA, et al. Variations in infection sites and mortality rates among patients in intensive care units with severe sepsis and septic shock in Japan. J Intensive Care. 2019;7:28.

20. Barochia AV, Cui X, Vitberg D, Suffredini AF, O'Grady NP, Banks SM, et al. Bundled care for septic shock: an analysis of clinical trials. Crit Care Med. 2010;38:668-78.

21. Andrews B, Semler MW, Muchemwa L, Kelly P, Lakhi S, Heimburger DC, et al. Effect of an early resuscitation protocol on in-hospital mortality among adults with sepsis and hypotension: a randomized clinical trial. JAMA. 2017;318:1233-40.

22. Deis AS, Whiles BB, Brown AR, Satterwhite CL, Simpson SQ. Three-hour bundle compliance and outcomes in patients with undiagnosed severe sepsis. Chest. 2018;153:39-45

23. Fujishima S, Gando S, Saitoh D, Mayumi T, Kushimoto S, Shiraishi S, et al. A multicenter, prospective evaluation of quality of care and mortality in Japan based on the Surviving Sepsis Campaign guidelines. J Infect Chemother 2014:20:115-20.

24. Pro Cl, Yealy DM, Kellum JA, Huang DT, Barnato AE, Weissfeld LA, et al. A randomized trial of protocol-based care for early septic shock. N Engl J Med. 2014;370:1683-93.

25. Investigators A, Group ACT, Peake SL, Delaney A, Bailey M, Bellomo R, et al. Goal-directed resuscitation for patients with early septic shock. N Engl J Med. 2014;371:1496-506

26. Hranjec $T$, Rosenberger $L H$, Swenson B, Metzger R, Flohr TR, Politano AD, et al. Aggressive versus conservative initiation of antimicrobial treatment in critically ill surgical patients with suspected intensive-care-unit-acquired infection: a quasi-experimental, before and after observational cohort study. Lancet Infect Dis. 2012:12:774-80.

27. Webb BJ, Sorensen J, Jephson A, Mecham I, Dean NC. Broad-spectrum antibiotic use and poor outcomes in community-onset pneumonia: a cohort study. Eur Respir J. 2019;54:1900057.

28. Abe T, Tokuda Y, Shiraishi A, Fujishima S, Mayumi T, Sugiyama T, et al. Inhospital mortality associated with the misdiagnosis or unidentified site of infection at admission. Crit Care. 2019;23:202.

\section{Publisher's Note}

Springer Nature remains neutral with regard to jurisdictional claims in published maps and institutional affiliations.

Ready to submit your research? Choose BMC and benefit from:

- fast, convenient online submission

- thorough peer review by experienced researchers in your field

- rapid publication on acceptance

- support for research data, including large and complex data types

- gold Open Access which fosters wider collaboration and increased citations

- maximum visibility for your research: over $100 \mathrm{M}$ website views per year

At BMC, research is always in progress.

Learn more biomedcentral.com/submissions 